\title{
Assembly of a Three-Dimensional Multitype Bronchiole Coculture Model Using Magnetic Levitation
}

\author{
Hubert Tseng, BS, Jacob A. Gage, BS, ${ }^{2,3}$ Robert M. Raphael, PhD, , Robert H. Moore, MD, ${ }^{1,2}$ \\ Thomas C. Killian, PhD, ${ }^{2,4}$ K. Jane Grande-Allen, PhD, and Glauco R. Souza, PhD ${ }^{2}$
}

A longstanding goal in biomedical research has been to create organotypic cocultures that faithfully represent native tissue environments. There is presently great interest in representative culture models of the lung, which is a particularly challenging tissue to recreate in vitro. This study used magnetic levitation in conjunction with magnetic nanoparticles as a means of creating an organized three-dimensional (3D) coculture of the bronchiole that sequentially layers cells in a manner similar to native tissue architecture. The 3D coculture model was assembled from four human cell types in the bronchiole: endothelial cells, smooth muscle cells (SMCs), fibroblasts, and epithelial cells (EpiCs). This study represents the first effort to combine these particular cell types into an organized bronchiole coculture. These cell layers were first cultured in 3D by magnetic levitation, and then manipulated into contact with a custom-made magnetic pen, and again cultured for $48 \mathrm{~h}$. Hematoxylin and eosin staining of the resulting coculture showed four distinct layers within the 3D coculture. Immunohistochemistry confirmed the phenotype of each of the four cell types and showed organized extracellular matrix formation, particularly, with collagen type I. Positive stains for CD31, von Willebrand factor, smooth muscle $\alpha$-actin, vimentin, and fibronectin demonstrate the maintenance of the phenotype for endothelial cells, SMCs, and fibroblasts. Positive stains for mucin-5AC, cytokeratin, and E-cadherin after 7 days with and without $1 \%$ fetal bovine serum showed that EpiCs maintained the phenotype and function. This study validates magnetic levitation as a method for the rapid creation of organized 3D cocultures that maintain the phenotype and induce extracellular matrix formation.

\section{Introduction}

A PRESENT CHALLENGE in biomedical research is the creation of in vitro organotypic models that faithfully represent native tissues, particularly, for the purposes of drug discovery. There is a large gap in complexity and in fidelity to native tissue properties between simple two-dimensional (2D) cell culture experiments and human tissues of interest, with animal models being the common bridge. However, there are many examples of drugs and treatments that show promise in vitro and in animal models, but do not show efficacy in humans, particularly, with treatments of respiratory disease. ${ }^{1-3}$ As such, there is a demand for organotypic models, that combine multiple cell types, mimic the tissue structure, and recreate native function. Such models would allow for cost-effective drug discovery, toxicological testing, and the study of other biological phenomena at the higher orders of complexity and fidelity necessary for translation to use in humans.
Toward this goal, this study attempts to create a layered, organized, coculture model of lung tissue consisting of four cell types. Many cell types exist in the body either in proximity to, or in direct contact with, another cell type, and intracellular cell signaling is important for regulating cell behavior. Cocultures leverage the presence and intracellular signaling between cell types, facilitating the study of heterotypic interactions, or for other uses, such as the promotion of stem cell differentiation. ${ }^{4}$ Although the need for cocultures clearly exists, there are limiting factors in creating complex and organized cocultures using existing cell culture techniques. Traditionally, cell culture has been performed on 2D surfaces, and the bulk of knowledge in cell biology today comes from discoveries made via conventional 2D cell culture. Attempts at 2D cocultures, which can be generalized as the transfer of signals between two exclusive cell monolayers, have included either the direct transfer of conditioned media between culture vessels, ${ }^{5,6}$ mixed cultures, ${ }^{6,7}$ a shared culture environment, ${ }^{8}$ or microfluidics. ${ }^{9-12}$

Departments of ${ }^{1}$ Bioengineering and ${ }^{4}$ Physics, Rice University, Houston, Texas.

${ }^{2}$ Nano3D Biosciences, Houston, Texas.

${ }^{3}$ Department of Pediatrics, Baylor College of Medicine, Houston, Texas. 
However, 2D cell cultures, in general, are limited in their capability to replicate the molecular gradients, ${ }^{13}$ substrate stiffness, ${ }^{14-16}$ and the spectrum of cell-cell and cell-matrix interactions ${ }^{17-19}$ observed in living tissue. As a result, there exists a need to create more representative environments using three-dimensional (3D) cell culture, toward which biological research has moved. Three-dimensional cell culture techniques offer the ability to control shape, structure, and biochemical environment, allowing researchers to conduct experiments at higher orders of complexity than those available in 2D. ${ }^{13,14,17,18,20,21}$ Three-dimensional cell culture techniques generally depend on promoting direct cell-cell interactions, using cell aggregation techniques like spheroids and pellet cultures, ${ }^{22,23}$ or cell-matrix interactions, either with protein gels ${ }^{24-26}$ or synthetic polymer scaffolds. ${ }^{27,28}$ When used for coculture, 3D approaches have varied, including the formation of spheroids with multiple cell types, ${ }^{29}$ encapsulated protein gels seeded with multiple cell types, ${ }^{30}$ or hybrid methods of 3D cultures and monolayers. ${ }^{31}$ In general, these methods have been successful in creating improved in vivo-like conditions, yet these methods often lack the spatial control necessary to organize cells into complex organotypic models that recapitulate tissue environments.

The model of choice for this study is the bronchiole, the downstream airway branches located proximal to the alveoli, which consists of four distinct cell types: epithelial cells (EpiCs), smooth muscle cells (SMCs), pulmonary fibroblasts (PFs), and pulmonary endothelial cells (PECs). ${ }^{32}$ There has been interest in creating lung coculture models because of potential applications to more complex studies of inflammatory and toxicological effects of particulate matter and inhalants, and airway remodeling. ${ }^{12,33,34}$ Cocultures have been regularly used in respiratory research; for example, porous membranes, such as Transwell membranes, between different cell types are used to promote cell-cell communication. ${ }^{12,35-38}$ Some models focused on both recreating the extracellular matrix environment and also culturing at the air-liquid interface, but those cultures were limited by the use of simple protein gel solutions, such as collagen or Matrigel, that were not truly representative of lung tissue and did not contain all four cell types. ${ }^{34,39-44}$ Thus, there is a need for a 3D model of the lung that mimics the native extracellular matrix with all of its cell types.

To create the lung coculture model reported here, we used magnetic levitation to assemble an organized structure. Magnetic levitation is based on the use of a nanoparticle assembly consisting of poly-L-lysine (PLL), magnetic iron oxide ( $\mathrm{MIO} ; \mathrm{Fe}_{3} \mathrm{O}_{4}$, magnetite), and gold nanoparticles that self-assemble into networks based on electrostatic interactions. ${ }^{45-49}$ Cellular uptake of the biocompatible nanoparticles renders the cell magnetic, allowing for magnetic manipulation of cells; in particular, cells in a culture dish can be levitated to the air-liquid interface with the application of a low-magnitude magnetic field (50$300 \mathrm{G})$, making it seemingly ideal for respiratory research applications. These cells interact, self-assemble, and prolif-

*A previous version of the nanoparticle assembly included bacteriophage displaying RGD-4C, which targets $\alpha \mathrm{v}$ integrins, but has since been replaced with PLL due to concerns of contamination with foreign biological molecules. erate without the need or influence of an artificial extracellular matrix; for example, magnetically levitated human glioblastoma cells showed greater proliferation and protein expression when they were magnetically levitated than when they were plated in 2 D. ${ }^{49,50}$ This technique has also been used to assemble models of cardiac SMCs, ${ }^{51}$ as well as cocultures of endothelial cells with adipose stem cells with in vivo-like vascular endothelial and adipose architecture. ${ }^{52}$ These attributes make magnetic levitation an attractive method for creating 3D multitype cocultures. For this study, as a proof of concept, we recreated the bronchiole wall from airway to circulation; this study is the first effort of its kind to coculture these particular four cell types together in a layered assembly, although it is not the first to put four cell types in coculture together. ${ }^{38,53,54}$ Lung cocultures were assembled and levitated using a sequential layered assembly technique to culture for 2 and 7 days. For 7-day cultures, $1 \%$ fetal bovine serum (FBS) was added to the medium to determine whether serum could be used for culture, while maintaining the EpiC phenotype. Histology and immunohistochemistry were performed to demonstrate the layered structure of the combined coculture, verify the phenotype of the constituent cell types, and track extracellular matrix formation.

\section{Materials and Methods}

\section{Cell culture}

All cell types in this study were primary human cells (ScienCell Research Laboratories) and cultured in a humidified environment $\left(37^{\circ} \mathrm{C}, 5 \% \mathrm{CO}_{2}\right)$ with media changes every other day.

For PEC (human pulmonary microvascular endothelial cells; ScienCell Research Laboratories) culture, before PECs were seeded, tissue culture polystyrene (TCPS) flasks and glass slides were first coated with a $2.5 \%$ gelatin (MP Biomedicals) solution in 1:1 (v/v) $\mathrm{dI} \mathrm{H}_{2} \mathrm{O}$ :phosphate-buffered saline (PBS, $\mathrm{pH}=7.4$ ) that was autoclaved before coating. ${ }^{55}$ The gelatin solution was sterilized for $1 \mathrm{~h}$ under ultraviolet light at room temperature. Before seeding with cells, an excess coating solution was aspirated from the surface. PECs were cultured in specialized media (ScienCell Research Laboratories) that contained 2\% FBS. These cells were expanded to their third passage before use.

Similarly, for EpiC (human bronchial epithelial cells; ScienCell Research Laboratories) culture, TCPS flasks and glass slides were coated with a $10 \mu \mathrm{g} / \mathrm{mL}$ PLL (Sigma-Aldrich) solution in PBS, for a final concentration of $2 \mu \mathrm{g} / \mathrm{cm}^{2}$ of cell culture surface area. The PLL solution was sterilized on the tissue culture surface for $1 \mathrm{~h}$ under ultraviolet light at room temperature. An excess coating solution was aspirated off the surface before the surface was seeded with cells. EpiCs were cultured in a specialized medium (ScienCell Research Laboratories) with no serum and expanded up to its third passage before use.

Both PFs (human pulmonary fibroblasts; ScienCell Research Laboratories) and SMCs (human tracheal smooth muscle cells; ScienCell Research Laboratories) were cultured on uncoated TCPS flasks using the Dulbecco's modified Eagle's medium (Mediatech) with 10\% bovine growth serum (Hyclone) for PFs, and specialized media for SMCs (ScienCell Research Laboratories) with 2\% FBS. For 2D 
immunohistochemistry, these two cell types were grown on PLL-coated glass slides, which were coated similarly to the glass slides coated for EpiCs. Both cell types were expanded up to their fifth passage before use.

\section{Magnetic levitation}

All 2D cultures of each cell type were converted into 3D cultures using the Bio-Assembler Kit (Nano3D Biosciences) (Fig. 1). Cells were grown in $2 \mathrm{D}$ to $75 \%-80 \%$ confluence, at which point, they were treated with an MIO-based nanoparticle assembly (NanoShuttle; Nano3D Biosciences) at a concentration of $8 \mu \mathrm{L} / \mathrm{cm}^{2}$ of cell culture surface area or $50 \mu \mathrm{L} / \mathrm{mL}$ media and incubated overnight. The next day, treated cells were enzymatically detached with trypsin and resuspended in either $2 \mathrm{~mL}$ of their own media in a Petri dish or $400 \mu \mathrm{L}$ per well in a 24 -well low-adhesion plate (Corning). A neodymium magnet was immediately placed on top of the plate to levitate the cells to the air-liquid interface (field strength: $300 \mathrm{G}$ for Petri dish, $50 \mathrm{G}$ for 24 -well plate). Threedimensional monotype cultures were levitated for $48 \mathrm{~h}$ before fixation.

\section{Coculture assembly}

After treatment overnight with NanoShuttle, SMCs, PFs, PECs, and EpiCs were individually detached from the flasks with trypsin and levitated concomitantly for $4 \mathrm{~h}$ in either a 24 -well plate $(200,000$ cells each cell type) or in a Petri dish ( 2 million cells each cell type) (Fig. 2). Once each cell type was levitated, a Teflon pen (0.1875" OD) with a rod magnet inside was used to pick up each cell type sequentially; first EpiCs, then SMCs, then PFs, and then PECs. This coculture of all four cell types is designated the bronchiole coculture (BCC). After assembly, the BCC, still attached to the pen, was submerged in serum-free EpiC media, and left to adhere on the pen for 4h. Afterward, the rod magnet was removed from the pen, and another magnet was placed underneath a well filled with fresh EpiC media to remove the BCC from the Teflon pen and hold it in place. The plate was covered and the magnet was moved from underneath to atop the well, re-levitating the BCC in EpiC media. The BCC was levitated and cultured for 2 and 7 days. Seven-day cultures were either cultured with and without 1\% FBS (see Supplementary Fig. S1; Supplementary Data are available online at www.liebertpub.com/tec).

\section{A. Grow cells to $70-80 \%$ confluence}

\section{B. Add nanoparticles and incubate overnight at $37^{\circ} \mathrm{C}$}

C. Trypsinize and resuspend
in media

\section{Distribute to petri dish (L) or 24 well plate $(\mathrm{R})$}

\section{E. Place neodymium magnet on top and levitate cells to air-liquid interface}
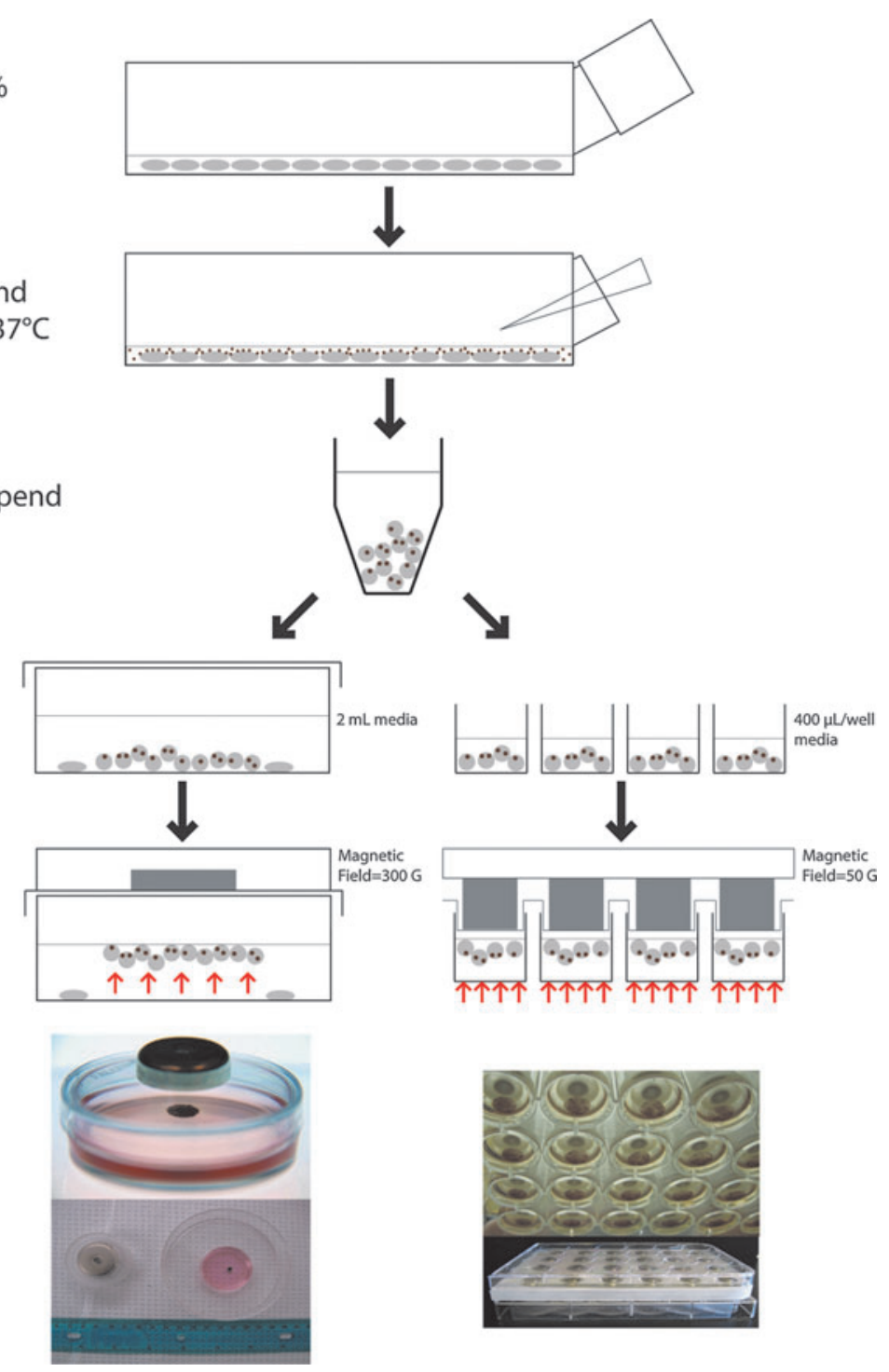

FIG. 1. Schematic of magnetic levitation in a Petri dish (left) and a 24-well plate (right). NanoShuttle is added to the culture vessel at $75 \%-80 \%$ confluence, and incubated with the NanoShuttle overnight for uptake. The next day, the cells are detached from the flask and resuspended in $2 \mathrm{~mL}$ of media in a Petri dish, or $400 \mu \mathrm{L}$ of media in a 24-well plate. The cells are levitated to the air-liquid interface by placing a neodymium magnet above the dish or well. Bottom from left to right: a levitated construct; a bird's eye view of the construct and its size; a 24-well plate with a customized magnetic lid for magnetic levitation; and levitated cell constructs in a 24-well plate. Color images available online at www.liebertpub.com/tec 
A. Grow each cell type to $70-80 \%$ confluence

B. Add nanoparticles, incubate overnight, trypsinize, and levitate for 4 hours before assembly
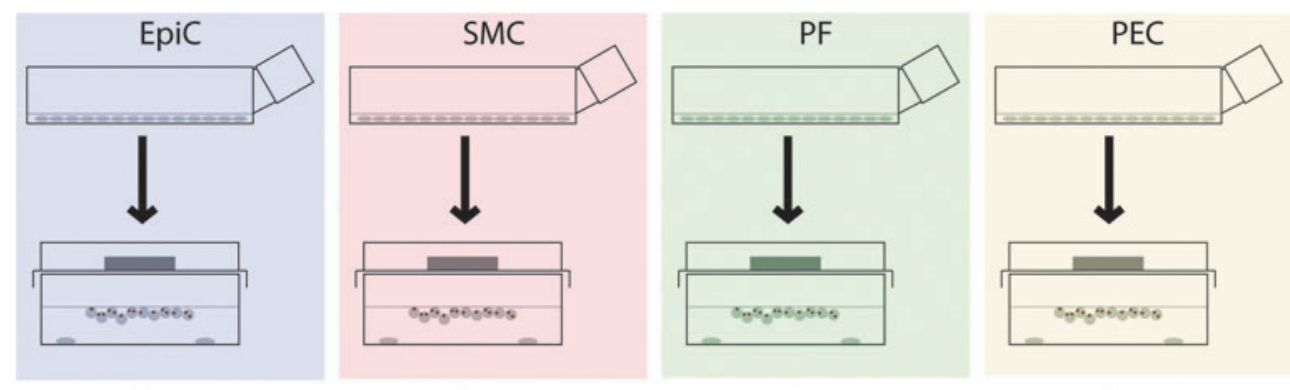

C. Sequentially pick up each cell type to assemble co-culture with a Teflon pen with magnet inside
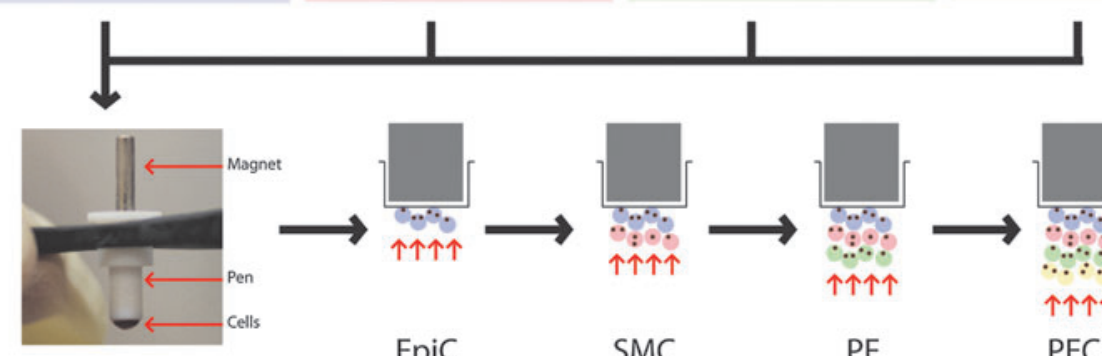

EpiC
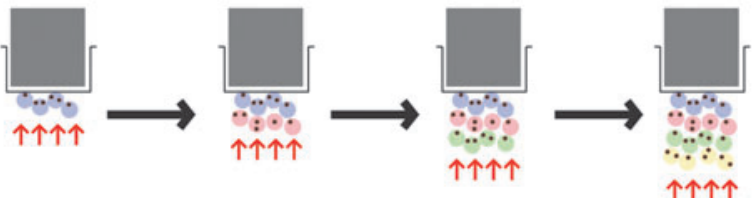

SMC

PF

PEC

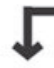

\section{Submerge Teflon pen with cells attached in EpiC media for 4 hours. After 4 hours, use magnet to remove co-culture off Teflon, and re-levitate to the air-liquid interface}
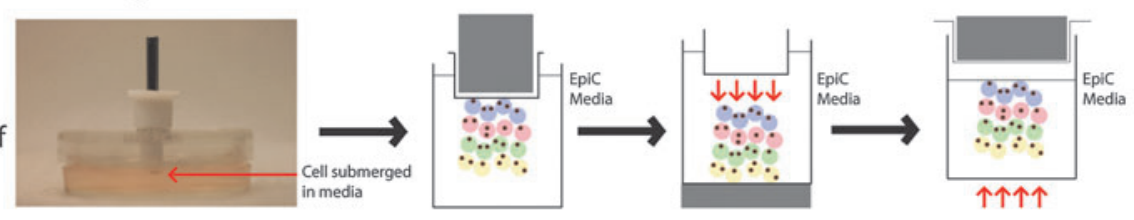

FIG. 2. Schematic of coculture assembly. Each individual cell type was grown to confluence and magnetically levitated to the air-liquid interface, with a cell number of either 2 million for Petri dishes, 200,000 for 24-well plates. After $4 \mathrm{~h}$ of levitation, a Teflon pen with a rod magnet inside was used to sequentially pick up the levitated cells: first epithelial cells (EpiCs), then smooth muscle cells (SMCs), pulmonary fibroblasts (PFs), and pulmonary endothelial cells (PECs). The pen, with the bronchiole coculture (BCC) attached to it, was submerged in EpiC media for $4 \mathrm{~h}$. The BCC was removed off the pen by removing the rod magnet and using a neodymium magnet underneath the dish or well to bring down the BCC. A magnet is placed above the dish or well to re-levitate the BCC to the air-liquid interface. Color images available online at www.liebertpub.com/tec

\section{Histology and immunohistochemistry}

After levitation, 3D cultures were fixed in $4 \%$ paraformaldehyde for a minimum of $5 \mathrm{~h}$. BCCs assembled in Petri dishes were paraffin-embedded for histology and immunohistochemistry. The same Teflon pen used for BCC assembly was used to pick up the BCC and keep it intact during processing. The BCCs were then gradually dehydrated in increasing concentrations of ethanol, and then xylene in 15-min steps, before being embedded in paraffin and sectioned according to standard procedures. Hematoxylin and eosin (H\&E) was used to stain cell nuclei (purple) and proteins (pink). BCCs assembled in 24-well plates were not embedded in paraffin and instead subjected to whole-mount immunohistochemistry.

Immunohistochemical staining was performed to detect particular phenotypic markers and extracellular matrix proteins. The phenotypic antigens investigated were smooth muscle $\alpha$-actin (Abcam) and vimentin (Millipore) for SMCs, fibronectin (Abcam) for PFs, and von Willebrand factor (Abcam) for PECs, mucin-5AC (Abcam), cytokeratin-19 (Abcam), and E-cadherin (Invitrogen) for EpiCs. The extracellular matrix antigens were collagen type I (Abcam) and laminin (Abcam). N-cadherin (Invitrogen) was also stained for.

Antigen retrieval was performed using a citrate buffer solution $(10 \times$ Antigen Decloaker; Biocare Medical) for $30 \mathrm{~min}$ at $80^{\circ} \mathrm{C}$. For laminin, sections were further pretreated using $600 \mathrm{U} / \mathrm{mL}$ bovine testicular hyaluronidase (Worthington Biochemical) at $37^{\circ} \mathrm{C}$ for $15 \mathrm{~min}$. After antigen retrieval, the sections with intracellular antigens were permeabilized using $0.2 \%$ Triton X-100 for $15 \mathrm{~min}$. All sections were blocked using a donkey serum buffer (DSB, 1\% donkey serum in PBS; Hyclone). Experimental sections were stained with the particular antibodies of interest at the manufacturer's recommended dilution in PBS with $1 \%$ bovine serum albumin. Negative controls were incubated with DSB during this time. All sections were washed and incubated with a fluorescent secondary antibody (AlexaFluor 488/555/633; Invitrogen) to the animal of the primary antibody. The sections were washed again and counterstained using 4',6-diamidino-2phenylindole (DAPI; KPL). For whole-mount immunohistochemistry, BCCs and 3D EpiC cultures were then stained similarly in a 96-well plate, but handled differently. Magnets were placed below the plate to magnetically hold the 3D cultures to the bottom, allowing for solutions to be added and removed. The Teflon pen for BCC assembly was used to transfer BCCs from well to well. For immunocytochemistry, 2D culture slides were fixed for $15 \mathrm{~min}$ in $4 \%$ paraformaldehyde, and then washed twice in PBS. Slides were then stained similarly to 3D sections starting with the permeabilization step. 

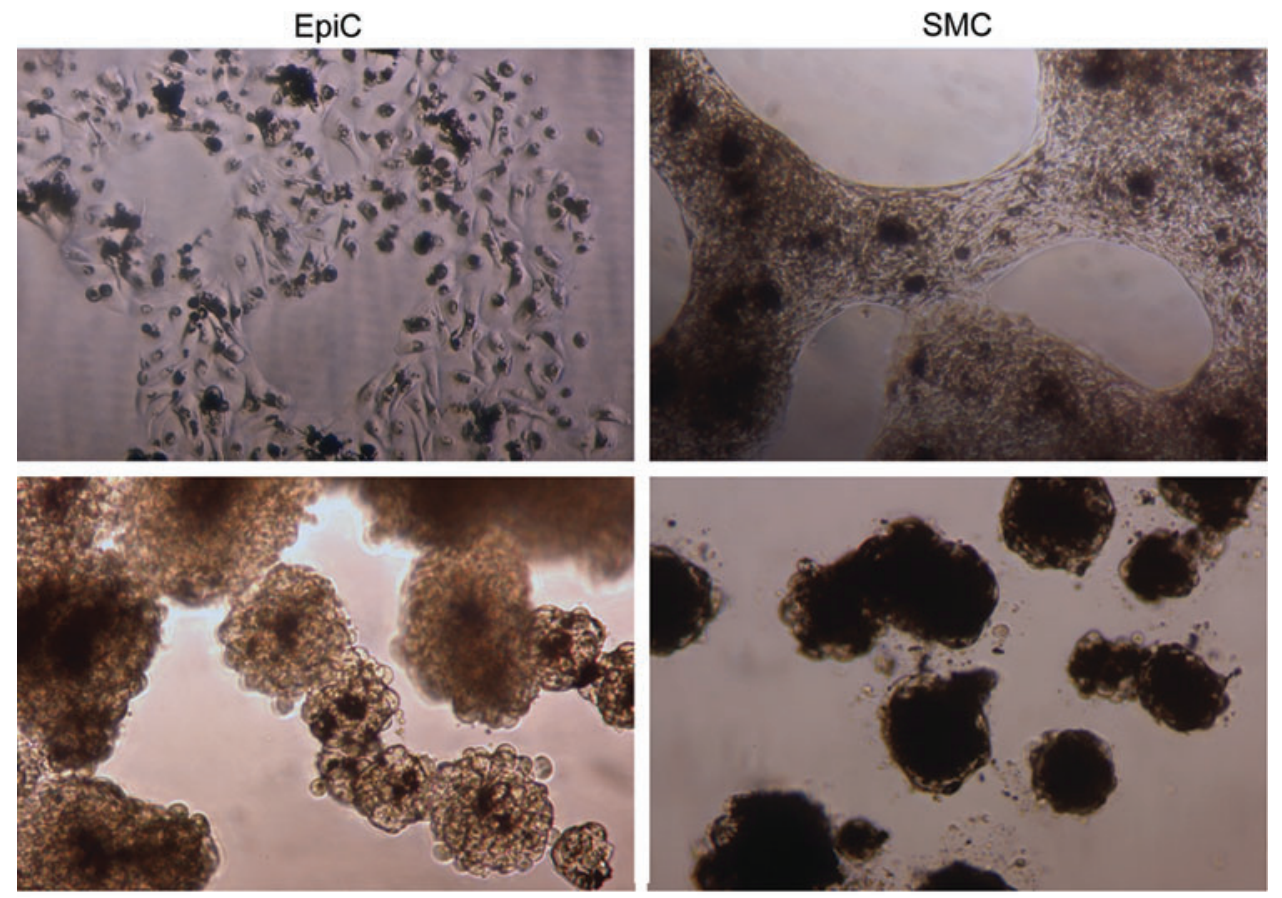

HPF

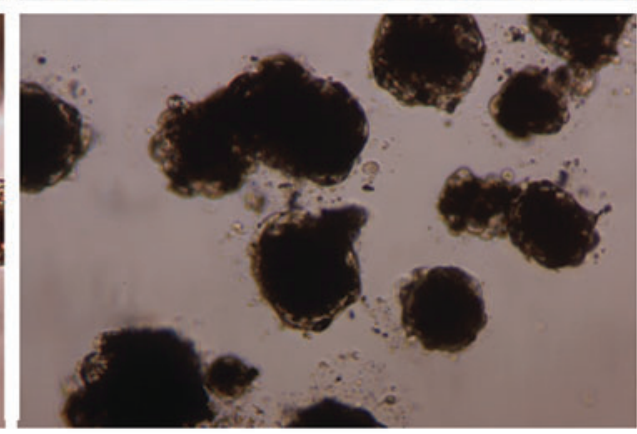

PEC
FIG. 3. Brightfield images of levitated 3D monotype cultures of EpiCs, SMCs, PFs, and PECs. Magnification $=10 \times$. Color images available online at www .liebertpub.com/tec
Fluorescent images were captured using an upright fluorescent microscope (Axio Imager Z2; Zeiss) and a confocal microscope (LSM 510 META NLO; Zeiss), while all other histology slides were imaged using an inverted light microscope (DM LS2; Leica Microsystems). Images were processed using ImageJ image processing software (NIH). All images of antigens stained in the same session with the same emission wavelength were processed identically by subtracting the background, and then enhancing the contrast based on the most intense image of the set. For DAPI counterstains, the same processing was conducted, except that the contrast was enhanced regardless of a secondary filter or staining session.

\section{Results}

The four cell types of the bronchiole (EpiCs, SMCs, PFs, and PECs) were levitated and assembled into the BCC containing all cell types. Both the application of NanoShuttle and exposure to the magnetic field had a minimal effect on the proliferation and inflammatory responses of all four cell types (see Supplementary Figs. S2-S4).

All cell types were individually levitated to the air-liquid interface successfully (Fig. 3). There were differences in structural integrity between the 3D monotype cultures; monotype PF and SMC cultures levitated in a Petri dish remained intact with the removal of the magnetic field, whereas monotype EpiC and PEC cultures levitated under the same conditions were less structurally robust and could break apart during routine handling. Both 3D cultures made in 24-well plates with smaller cell numbers (200,000 cells) and lower media volumes $(400 \mu \mathrm{L})$, as well as cultures made in Petri dishes with larger cell numbers $(2,000,000$ cells) and higher volumes $(4 \mathrm{~mL})$, did not lose the structure with the removal of the magnetic field. Thus, all four cell types were levitated together for $4 \mathrm{~h}$ before assembly of the coculture.
Regardless, each monotype 3D cell culture could be picked up using the magnetic pen and transferred with minimal difficulty. The BCC maintained its structure when the magnetic field was removed and when transferred.

The BCC showed regional differences in the cell morphology and extracellular matrix content (Fig. 4). As demonstrated by the color of the H\&E stain, cell morphology, and the distribution of NanoShuttle, it was possible to see the distribution of the four cell types, which were present in a relatively layered, organized fashion, starting from one surface to the other. There were no clear boundaries between layers, which suggest that there was integration between the different cell types at the interfaces. As assembled, the PECs formed a thin layer at one surface of the BCC and was characterized by a high NanoShuttle density and elongated

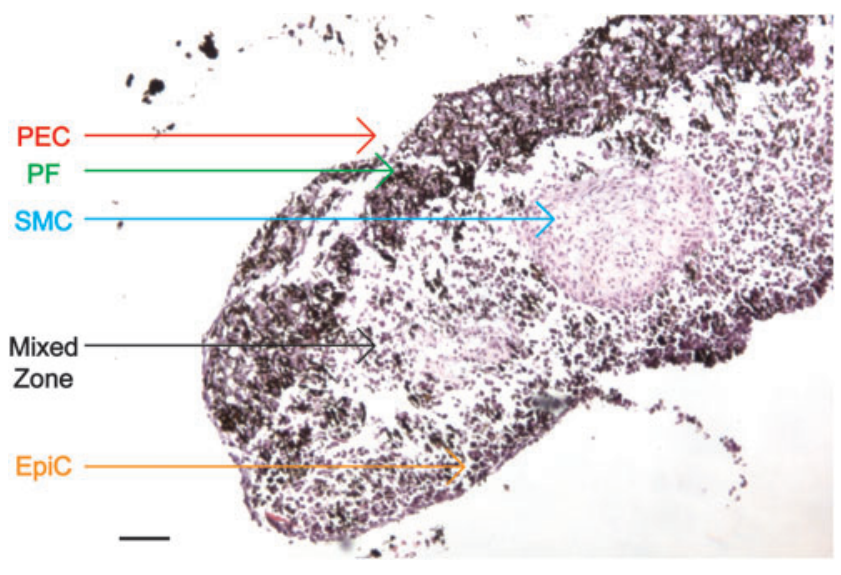

FIG. 4. H\&E staining of the BCC. Note the organization in the BCC that corresponds to four different cell types, as well as a mixed zone within the center of the BCC. Scale bar $=100 \mu \mathrm{m}$. H\&E, hematoxylin and eosin. Color images available online at www.liebertpub.com/tec 
cell morphologies. Above the PEC layer were the PFs, which were apparent with a darker magenta stain, and formed a thick layer of the extracellular matrix. The next layer was a mixed zone, in which SMCs, colored light pink, aggregated into masses showing highly elongated cell morphologies and low NanoShuttle density, and was surrounded by globular cells, which appeared to be PFs by their color. At the opposite surface of the BCC were the EpiCs, which formed a thin one- to two-cell thick layer.

Immunohistochemical staining for phenotypic markers in each cell type was positive in both the $3 \mathrm{D}$ cultures of the individual cell types and the BCC (Fig. 5). The positive

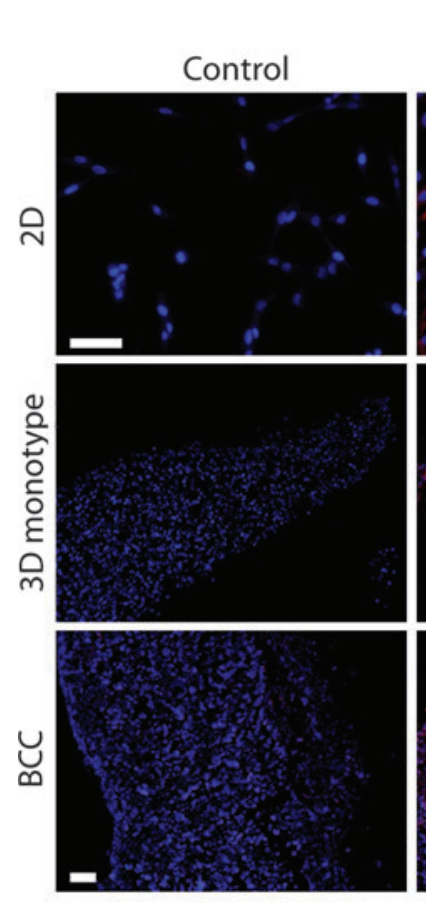

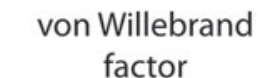
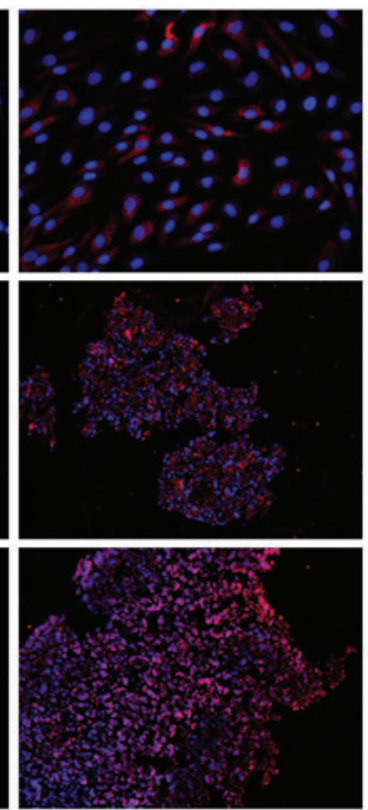

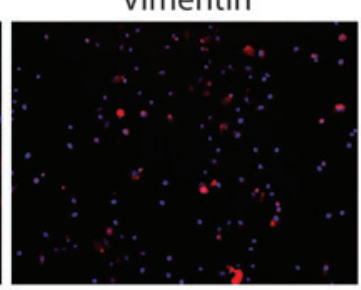

\section{Smooth muscle}

a-actin
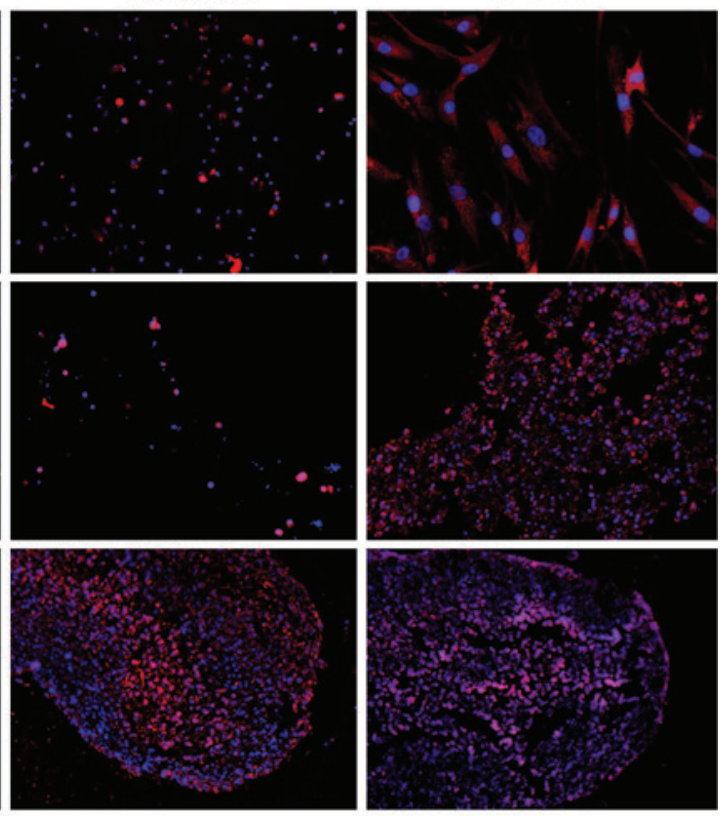
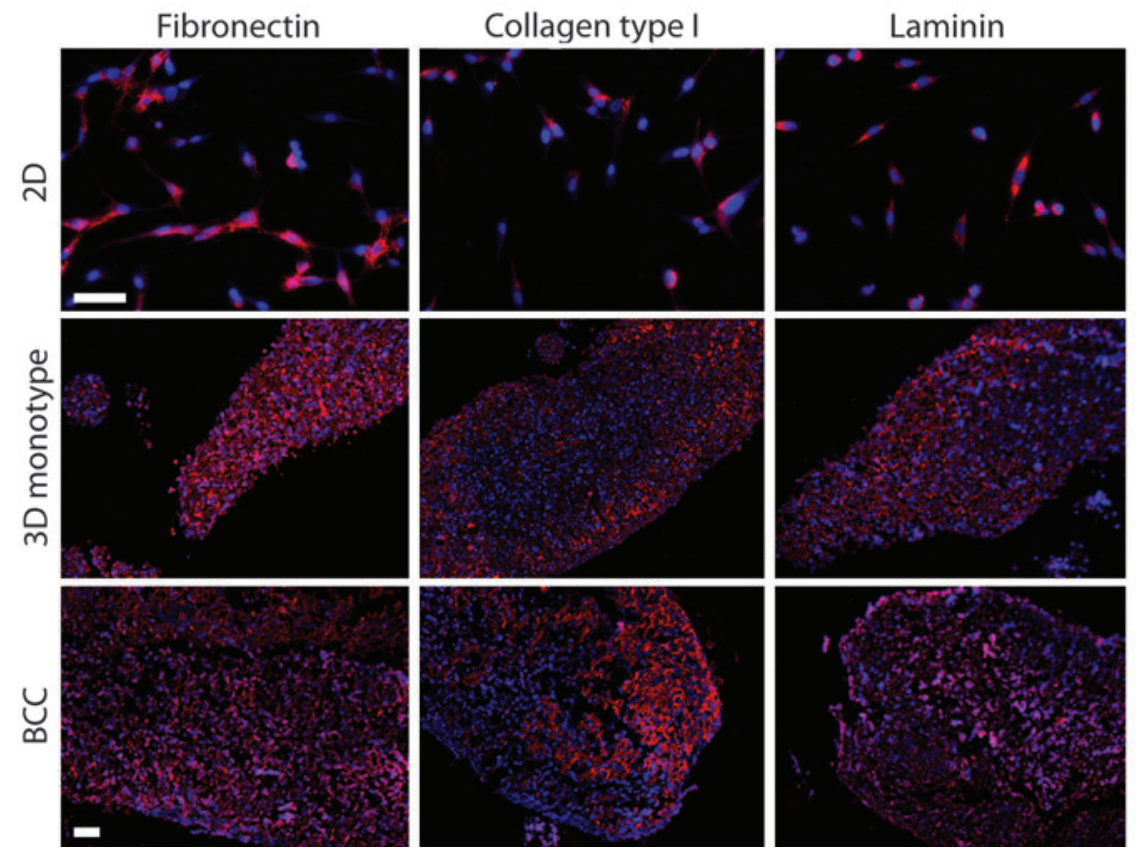

FIG. 5. Immunohistochemical stains (red) for von Willebrand factor, smooth muscle $\alpha$-actin, fibronectin, vimentin, collagen type I, and laminin of the BCC, and three-dimensional (3D) mono-type cultures and two-dimensional (2D) cultures (PECs for the von Willebrand factor, SMCs for smooth muscle $\alpha$-actin and vimentin, PFs for fibronectin, collagen type I, and laminin). Nuclei were counterstained with DAPI (blue). This BCC was assembled in a Petri dish with 2 million cells per cell layer, and fixed after 2 days of culture. Phenotypic markers follow an organized structure within the BCC, with some staining of von Willebrand factor in the center, suggesting migration of PECs to the center. The BCC demonstrates organized extracellular matrix formation, with collagen type I, in particular, forming a thick layer at one edge. Scale bar=100 $\mu \mathrm{m}$. DAPI, 4',6diamidino-2-phenylindole. Color images available online at www.liebertpub.com/tec 
phenotypic stains generally followed the organized distribution of the cells shown in the H\&E stain. Interestingly, von Willebrand factor, which is characteristically produced by PECs, positively stained in the center of the BCC. Compared to the staining of cells grown in 2D, the cells in 3D cultures appeared smaller in size, more globular in shape, and more densely packed (Fig. 5). BCCs and 3D EpiC cultures were also positive for epithelial phenotypic markers such as mucin-5AC, cytokeratin, and E-cadherin, as well as N-cadherin after 2 days, and 7 days of culture with and without 1\% FBS (Fig. 6). Monotype 3D EpiC cultures were generally lacking in structure and cellularity compared to the other cell types. The BCCs also stained positive for Ki67 (see Supplementary Fig. S5).

Extracellular matrix components, such as collagen type I and laminin, also stained positively in the BCC and in 3D monotype culture, as opposed to the 2D stains, in which these matrix proteins were faintly stained and localized with the cells (Fig. 5). In the BCC, collagen type I was in abundance within the PF layer underneath the PEC layer. In contrast, fibronectin and laminin stained uniformly throughout the thickness of the BCC. Monotype 3D cultures alone were able to produce the extracellular matrix, as demonstrated by the positive stain for collagen type I in PF cultures (Fig. 5), as well as positive laminin stains in PF and SMC cultures with under $6 \mathrm{~h}$ of levitation (see Supplementary Fig. S6). This positive laminin stain in 3D cultures stands in stark contrast to the nonpermeabilized immunocytochemical stains in 2D, where laminin did not stain positively until 48 h (see Supplementary Fig. S7).

\section{Discussion}

In this study, we used magnetic levitation to construct a multitype 3D coculture model of the bronchiole wall. Magnetic levitation was recently developed as a simple and

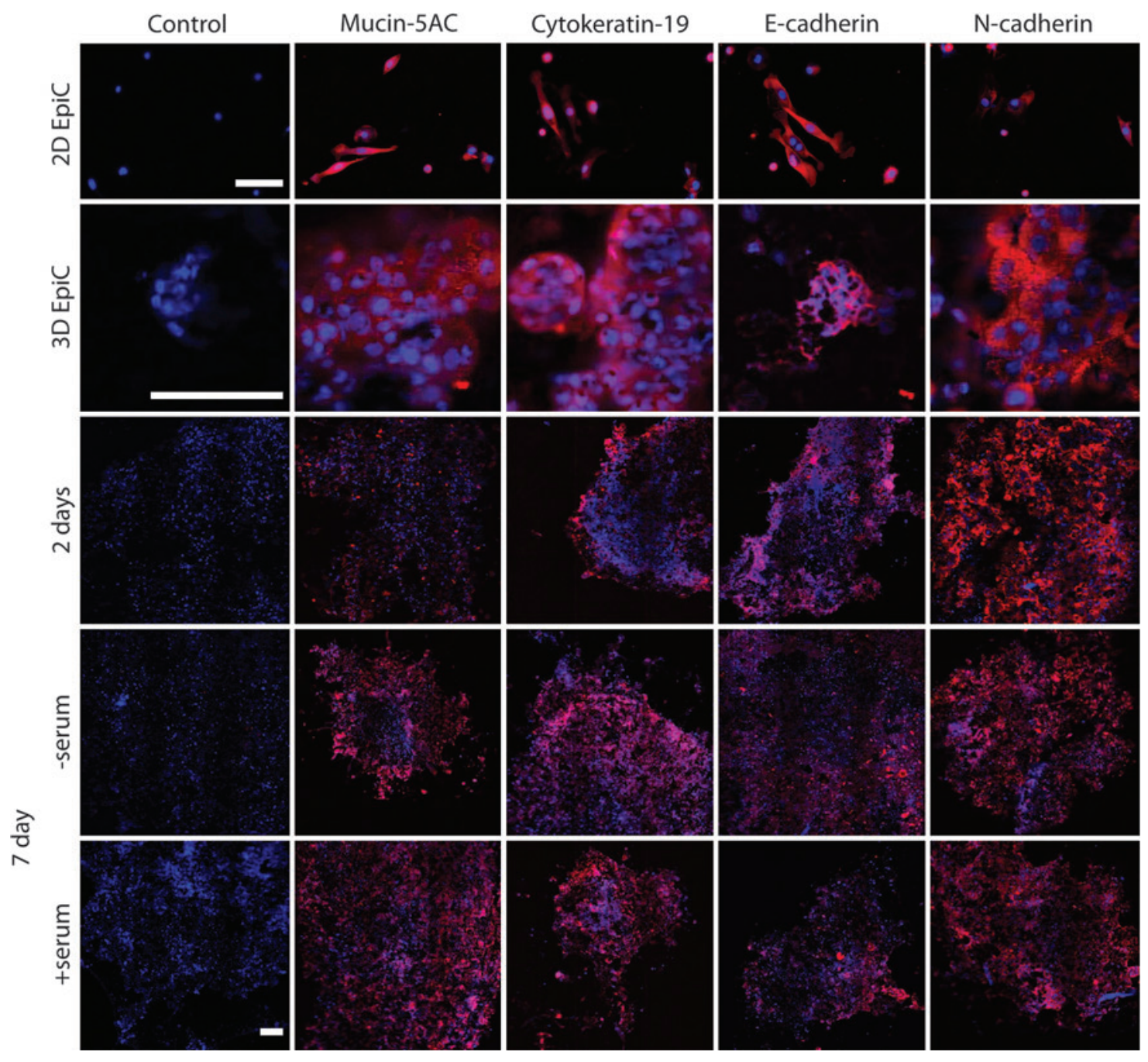

FIG. 6. Immunohistochemical stains (red) for mucin, cytokeratin, E-cadherin, and N-cadherin of the BCC after 2 days, 7 days with and without fetal bovine serum, and 3D mono-type cultures and 2D cell culture of EpiCs after 2 days. Nuclei were counterstained with DAPI (blue). These BCCs were assembled in a 24 -well plate with 200,000 cells per cell layer. The positive stains for these markers demonstrate the maintenance of the epithelial cell phenotype and function after 1 week of culture, as well as the ability to maintain the epithelial phenotype with serum. There is a stark difference in the staining intensity and pattern of mucin between 2D and 3D cultures. Positive staining for N-cadherin demonstrates the cell-cell interaction within the BCC. Scale bar $=100 \mu \mathrm{m}$. Color images available online at www.liebertpub.com/tec 
flexible method for creating 3D cell cultures. ${ }^{49}$ The advantages of using magnetic levitation to create cocultures are numerous: (1) the use of PLL in the NanoShuttle makes this method broadly applicable to most cell types, (2) the small amount of additional supplies (nanoparticle assembly, neodymium magnets) to create these cultures makes it a costeffective transition between 2D and 3D cell cultures, (3) magnetic manipulation allows for spatial control, (4) better proliferation and in vivo-like protein expression than $2 \mathrm{D}$ cultures, ${ }^{49}$ and (5) easy postculture manipulation and wholemount immunohistochemistry by holding cultures to the well bottom.

In this work, we sought to broaden the application of magnetically levitated 3D cell cultures to construct a 3D BCC of four cell types (EpiCs, SMCs, PFs, and PECs). This study represents the first effort to combine these particular cell types together in a layered coculture. It took $\sim 8 \mathrm{~h}$ to assemble the BCC from 2D individual cell cultures. Immunohistochemical and histological staining showed that the phenotype of each cell type was maintained, and that the various cell types were organized in a structure similar to the native bronchiole. Mucus production and secretion by EpiCs, as shown by positive mucin-5AC staining, was maintained in the BCC after 7 days, with and without 1\% FBS, indicating the maintenance of the epithelial function. In addition, the positive stains for collagen type I, fibronectin, and laminin demonstrated that the BCC induces the production of a densely organized extracellular matrix, in contrast to the negligible amount of extracellular matrix produced in 2D.

Extracellular matrix production was highly organized in the BCC, particularly collagen type I, which is abundant in the native lung ${ }^{56}$ and formed a thick layer close to one surface of the BCC. Monotype 3D cultures of PFs and SMCs also formed organized laminin structures within hours (see Supplementary Fig. S6). This pattern suggests that PFs and SMCs actively contribute to extracellular matrix synthesis and organization. Indeed, a comparison of permeabilized and nonpermeabilized immunohistochemical stains of 3D PF and SMC cultures appears to suggest that these cells are not just extruding existing intracellular laminin into the extracellular space, but actively producing it (see Supplementary Fig. S8). Thus, this model has great potential for research into airway remodeling associated with asthma. ${ }^{57-59}$ That these cell types alone can create an intact matrix merits further investigation into the genesis and progression of matrix formation in the lung, and the importance of their interactions with other cell types in this process. It is also worth noting that the discovery of the potential of PFs and SMCs alone to generate the extracellular matrix would not have been possible in $2 \mathrm{D}$, since in $2 \mathrm{D}$ stains, positive collagen type I and laminin stains localized around the cell. In addition, the organic production of the extracellular matrix within the BCC highlights the advantage of magnetically levitated 3D culture of achieving a complex model of the lung without the use of protein gel substrates. Indeed, the ability of this method to induce the matrix and basement membrane formation validates the utility of magnetic levitation in creating more representative tissue environments.

BCCs assembled in a 24-well plate demonstrated basic epithelial phenotype and function by the positive wholemount immunohistochemical stains of mucin-5AC, cytokeratin-19, and E-cadherin after 7 days. Mucus production was much higher in the BCC than monotype 3D and 2D EpiC cultures. In addition, 2D EpiC cultures were observed to have a lower cellularity, and were more delicate and difficult to handle. That the BCC had a stronger expression of EpiC phenotypic markers than monotype 3D EpiC cultures suggests the need for at least a second cell type to coculture with EpiCs to maintain cellularity and their phenotype. Indeed, EpiCs have been shown to be modulated by fibroblasts. ${ }^{60,61}$ Together, these results demonstrate the value of this model in creating representative models of the bronchiole.

A design limitation to cocultures is the choice of media, which is critical to ensure the maintenance of all cell phenotypes and their survival, as EpiCs are the most sensitive cell types to serum. However, 7-day cultures with 1\% FBS added maintained the EpiC phenotype, suggesting that longterm cultures with serum can be accomplished. Future research into the BCC or a model that utilizes the technique presented must focus on the optimization of media and serum concentration for the purpose of promoting the growth of all cell types, while maintaining phenotypes.

This study also introduced the use of a 24-well plate for magnetic levitation instead of the Petri dish. ${ }^{49}$ Assembling the BCC in a Petri dish required large cell numbers, and whose size did not always translate into structurally sound 3D cultures, as evidenced by the breakdown of the PEC and EpiC culture structure with the removal of the magnetic field. As a result, the timing of assembly was affected, which was set to balance the integration of the cell layers with the levitated cell yield. However, in the 24-well plate design presented, less cells, less media volume, and a weaker magnetic field allowed for the creation of structurally sound structures. This simple design could be further expanded to utilize other size well plates, like 96-well plates, allowing for high-throughput research.

This magnetically levitated model compares well with the most advanced lung tissue model in literature, which was a three-cell-type model of lung PFs, SMCs, and small airway EpiCs that was designed to study airway remodeling. ${ }^{34}$ Briefly, this previous model employed cylindrical collagen gels embedded with PFs, and then seeded with bronchial EpiCs on the lumen side and SMCs on the exterior side. This coculture was then loaded into a bioreactor to mimic mechanical stimulation and air environment. The collagen gel construct took 2-3 weeks to assemble, and was cultured for over 60 days, during which time, the PF phenotype transitioned from contractile to quiescent. ${ }^{34}$ Both this previous model and the BCC were exposed to the air-liquid interface; for the collagen gel coculture, a bioreactor pushed air through and across the surface of the collagen gel, while this current study used a magnetic field to bring the BCC to the air-liquid interface. The main difference between the two models lies in the time and method of assembly. Rather than seeding surfaces and requiring long incubation, magnetic levitation allowed for a modular layered approach to coculture assembly that took only hours. The BCC demonstrated relevant extracellular matrix formation and maintained the cell phenotype in significantly less time and without the need for a protein gel substrate. Previous studies of magnetically levitated 3D cultures demonstrated that 3D cultures of human glioblastoma cells could be maintained for at least 8 days, ${ }^{49}$ with longer culture times past 7 days possible. Another distinction between this BCC and the collagen 
gel model is the inclusion of PECs, whose role in promoting angiogenesis in asthma is a potential research topic of interest. ${ }^{59}$ Overall, the BCC assembled in this study is a viable 3D coculture of the four cell types of the lung that can be easily assembled and studied within a short period of time.

It is also important to note that this study serves as a proof of concept, in which the BCC can be made with magnetic levitation. The BCC assembled here maintains the basic EpiC phenotype and function, as indicated by the positive stains for mucin-5AC, cytokeratin, and E-cadherin, and induces extracellular matrix formation, as shown by the presence of collagen type I and laminin. Further studies will need to be conducted to optimize cellular behavior, before progressing toward more complex mechanistic studies using this model.

In conclusion, the presented data demonstrate the utility of magnetic levitation as a tool to create layered and organized cocultures. The BCC was assembled in hours, and exhibited extracellular matrix formation and maintenance of phenotype up to 7 days. The BCC could be used in the future to investigate inflammatory responses, angiogenesis, and airway remodeling. Magnetically levitated cocultures, in general, are simple to assemble, and their versatility bodes well for future applications of 3D cell culture in other complex tissue systems.

\section{Acknowledgments}

The authors would like to acknowledge Carly Filgueira, $\mathrm{PhD}$, and Chris Bertucci, Nano3D Biosciences, for their help with magnetic levitation; Daniel Harrington, $\mathrm{PhD}$, the Rice University, for his help with microscopy; and Joel Moake, $\mathrm{PhD}$, and Nancy Turner, PhD, the Rice University, for preliminary studies using aliquots of their von Willebrand factor antibody. Richard Clark, $\mathrm{PhD}$, and Joseph Alcorn, $\mathrm{PhD}$, the University of Texas Medical School at Houston, for their expertise in respiratory research and advice regarding the current study. This study was funded, in part, by the National Science Foundation (NSF) Small Business Innovation Research Award Phase I (0945954) and Phase II (1127551) from NSF IIP Division of Industrial Innovation and Partnerships and the State of Texas Emerging Technology Fund.

\section{Author Disclosure Statement}

The University of Texas M. D. Anderson Cancer Center (UTMDACC) and Rice University, along with their researchers, have filed patents on the technology and intellectual property reported here. If licensing or commercialization occurs, the researchers are entitled to standard royalties. Glauco R. Souza, Robert M. Raphael, and T.C. Killian have equity in Nano3D Biosciences, Inc. UTMDACC and Rice University manage the terms of these arrangements in accordance to their established institutional conflict-of-interest policies.

\section{References}

1. Krug, N., and Rabe, K.F. Animal models for human asthma: the perspective of a clinician. Curr Drug Targets 9, 438, 2008.

2. Kawut, S.M., Bagiella, E., Lederer, D.J., Shimbo, D., Horn, E.M., Roberts, K.E., Hill, N.S., Barr, R.G., Rosenzweig, E.B., Post, W., Tracy, R.P., Palevsky, H.I., Hassoun, P.M., and Girgis, R.E. Randomized clinical trial of aspirin and sim- vastatin for pulmonary arterial hypertension: ASA-STAT. Circulation 123, 2985, 2011.

3. Forsythe, P. Probiotics and lung diseases. Chest 139, 901, 2011.

4. Stacey, G.N., Cobo, F., Nieto, A., Talavera, P., Healy, L., and Concha, A. The development of "feeder" cells for the preparation of clinical grade hES cell lines: challenges and solutions. J Biotechnol 125, 583, 2006.

5. Thio, C.L., Waxman, S.G., and Sontheimer, H. Ion channels in spinal cord astrocytes in vitro. III. Modulation of channel expression by coculture with neurons and neuronconditioned medium. J Neurophysiol 69, 819, 1993.

6. Ellington, J.A., Carney, E.W., Farrell, P.B., Simkin, M.E., and Foote, R.H. Bovine 1-2-cell embryo development using a simple medium in three oviduct epithelial cell coculture systems. Biol Reprod 43, 97, 1990.

7. Snyder, E.Y., Deitcher, D.L., Walsh, C., Arnold-Aldea, S., Hartwieg, E.A., and Cepko, C.L. Multipotent neural cell lines can engraft and participate in development of mouse cerebellum. Cell 68, 33, 1992.

8. Haller, D., Holt, L., Parlesak, A., Zanga, J., Bäuerlein, A., Sartor, R.B., and Jobin, C. Differential effect of immune cells on non-pathogenic Gram-negative bacteria-induced nuclear factor-kappaB activation and pro-inflammatory gene expression in intestinal epithelial cells. Immunology 112, 310, 2004.

9. Montanez-Sauri, S.I., Sung, K.E., Puccinelli, J.P., Pehlke, C., and Beebe, D.J. Automation of three-dimensional cell culture in arrayed microfluidic devices. J Lab Autom 16, 171, 2011.

10. Marimuthu, M., and Kim, S. Microfluidic cell coculture methods for understanding cell biology, analyzing bio/ pharmaceuticals, and developing tissue constructs. Anal Biochem 413, 81, 2011.

11. Zhang, C., Zhao, Z., Abdul Rahim, N.A., Van Noort, D., and $\mathrm{Yu}, \mathrm{H}$. Towards a human-on-chip: culturing multiple cell types on a chip with compartmentalized microenvironments. Lab Chip 9, 3185, 2009.

12. Huh, D., Matthews, B.D., Mammoto, A., Montoya-Zavala, M., Hsin, H.Y., and Ingber, D.E. Reconstituting organ-level lung functions on a chip. Science 328, 1662, 2010.

13. Zhang, S. Beyond the Petri dish. Nat Biotechnol 22, 151, 2004.

14. Griffith, L.G., and Swartz, M.A. Capturing complex 3D tissue physiology in vitro. Nat Rev Mol Cell Biol 7, 211, 2006.

15. Peyton, S.R., Kim, P.D., Ghajar, C.M., Seliktar, D., and Putnam, A.J. The effects of matrix stiffness and RhoA on the phenotypic plasticity of smooth muscle cells in a 3-D biosynthetic hydrogel system. Biomaterials 29, 2597, 2008.

16. Pedersen, J.A., and Swartz, M.A. Mechanobiology in the third dimension. Ann Biomed Eng 33, 1469, 2005.

17. Cukierman, E., Pankov, R., Stevens, D.R., and Yamada, K.M. Taking cell-matrix adhesions to the third dimension. Science 294, 1708, 2001.

18. Pampaloni, F., Reynaud, E.G., and Stelzer, E.H.K. The third dimension bridges the gap between cell culture and live tissue. Nat Rev Mol Cell Biol 8, 839, 2007.

19. Kleinman, H.K., Philp, D., and Hoffman, M.P. Role of the extracellular matrix in morphogenesis. Curr Opin Biotechnol 14, 526, 2003.

20. Abbott, A. Biology's new dimension. Nature 424, 870, 2003.

21. Atala, A. Engineering tissues, organs and cells. J Tissue Eng Regen Med 1, 83, 2007.

22. Hirschhaeuser, F., Menne, H., Dittfeld, C., West, J., MuellerKlieser, W., and Kunz-Schughart, L.A. Multicellular tumor 
spheroids: an underestimated tool is catching up again. J Biotechnol 148, 3, 2010.

23. Bernstein, P., Dong, M., Corbeil, D., Gelinsky, M., Günther, K.-P., and Fickert, S. Pellet culture elicits superior chondrogenic redifferentiation than alginate-based systems. Biotechnol Prog 25, 1146, 2009.

24. Bell, E., Ivarsson, B., and Merrill, C. Production of a tissuelike structure by contraction of collagen lattices by human fibroblasts of different proliferative potential in vitro. Proc Natl Acad Sci U S A 76, 1274, 1979.

25. Shi, Y., and Vesely, I. Fabrication of mitral valve chordae by directed collagen gel shrinkage. Tissue Eng 9, 1233, 2003.

26. Nirmalanandhan, V.S., Duren, A., Hendricks, P., Vielhauer, G., and Sittampalam, G.S. Activity of anticancer agents in a three-dimensional cell culture model. Assay Drug Dev Technol 8, 581, 2010.

27. Cuchiara, M.P., Allen, A.C.B., Chen, T.M., Miller, J.S., and West, J.L. Multilayer microfluidic PEGDA hydrogels. Biomaterials 31, 5491, 2010.

28. Xu, X., and Prestwich, G.D. Inhibition of tumor growth and angiogenesis by a lysophosphatidic acid antagonist in an engineered three-dimensional lung cancer xenograft model. Cancer 116, 1739, 2010.

29. Chambers, K.F., Pearson, J.F., Aziz, N., O'Toole, P., Garrod, D., and Lang, S.H. Stroma regulates increased epithelial lateral cell adhesion in 3D culture: a role for actin/cadherin dynamics. PLoS One 6, e18796, 2011.

30. Butcher, J.T., and Nerem, R.M. Valvular endothelial cells regulate the phenotype of interstitial cells in co-culture: effects of steady shear stress. Tissue Eng 12, 905, 2006.

31. Van Hoof, D., Braam, S.R., Dormeyer, W., Ward-van Oostwaard, D., Heck, A.J.R., Krijgsveld, J., and Mummery, C.L. Feeder-free monolayer cultures of human embryonic stem cells express an epithelial plasma membrane protein profile. Stem Cells 26, 2777, 2008.

32. Mescher, A.L. The respiratory system. In: Mescher, A.L. Junqueira's Basic Histology: Text \& Atlas. New York, NY: McGraw-Hill Lange, 2009, pp. 298-315.

33. Klein, S.G., Hennen, J., Serchi, T., Blömeke, B., and Gutleb, A.C. Potential of coculture in vitro models to study inflammatory and sensitizing effects of particles on the lung. Toxicol In Vitro 25, 1516, 2011.

34. Miller, C., George, S., and Niklason, L. Developing a tissueengineered model of the human bronchiole. J Tissue Eng Regen Med 4, 619, 2010.

35. Pohl, C., Papritz, M., Moisch, M., Wübbeke, C., Hermanns, M.I., Uboldi, C., Dei-Anang, J., Mayer, E., Kirkpatrick, C.J., and Kehe, K. Acute morphological and toxicological effects in a human bronchial coculture model after sulfur mustard exposure. Toxicol Sci 112, 482, 2009.

36. Kasper, J., Hermanns, M.I., Bantz, C., Maskos, M., Stauber, R., Pohl, C., Unger, R.E., and Kirkpatrick, J.C. Inflammatory and cytotoxic responses of an alveolar-capillary coculture model to silica nanoparticles: comparison with conventional monocultures. Part Fibre Toxicol 8, 6, 2011.

37. Kuo, P.-L., Hsu, Y.-L., Huang, M.-S., Chiang, S.-L., and Ko, Y.-C. Bronchial epithelium-derived IL-8 and RANTES increased bronchial smooth muscle cell migration and proliferation by Krüppel-like factor 5 in areca nut-mediated airway remodeling. Toxicol Sci 121, 177, 2011.

38. Alfaro-Moreno, E., Nawrot, T.S., Vanaudenaerde, B.M., Hoylaerts, M.F., Vanoirbeek, J.A., Nemery, B., and Hoet, P.H.M. Co-cultures of multiple cell types mimic pulmonary cell communication in response to urban PM10. Eur Respir J 32, 1184, 2008.

39. Thompson, H.G.R., Mih, J.D., Krasieva, T.B., Tromberg, B.J., and George, S.C. Epithelial-derived TGF-beta2 modulates basal and wound-healing subepithelial matrix homeostasis. Am J Physiol Lung Cell Mol Physiol 291, L1277, 2006.

40. Choe, M.M., Sporn, P.H.S., and Swartz, M.A. An in vitro airway wall model of remodeling. Am J Physiol Lung Cell Mol Physiol 285, L427, 2003.

41. Choe, M.M., Tomei, A.A., and Swartz, M.A. Physiological 3D tissue model of the airway wall and mucosa. Nat Protoc 1, 357, 2006.

42. Choe, M.M., Sporn, P.H.S., and Swartz, M.A. Extracellular matrix remodeling by dynamic strain in a three-dimensional tissue-engineered human airway wall model. Am J Respir Cell Mol Biol 35, 306, 2006.

43. Chakir, J., Pagé, N., Hamid, Q., Laviolette, M., Boulet, L.P., and Rouabhia, M. Bronchial mucosa produced by tissue engineering: a new tool to study cellular interactions in asthma. J Allergy Clin Immunol 107, 36, 2001.

44. Asaithamby, A., Hu, B., Delgado, O., Ding, L.-H., Story, M.D., Minna, J.D., Shay, J.W., and Chen, D.J. Irreparable complex DNA double-strand breaks induce chromosome breakage in organotypic three-dimensional human lung epithelial cell culture. Nucleic Acids Res 39, 5474, 2011.

45. Hajitou, A., Trepel, M., Lilley, C.E., Soghomonyan, S., Alauddin, M.M., Marini, F.C., Restel, B.H., Ozawa, M.G., Moya, C.A., Rangel, R., Sun, Y., Zaoui, K., Schmidt, M., Von Kalle, C., Weitzman, M.D., Gelovani, J.G., Pasqualini, R., and Arap, W. A hybrid vector for ligand-directed tumor targeting and molecular imaging. Cell 125, 385, 2006.

46. Arap, W., Pasqualini, R., and Ruoslahti, E. Cancer treatment by targeted drug delivery to tumor vasculature in a mouse model. Science 279, 377, 1998.

47. Souza, G.R., Christianson, D.R., Staquicini, F.I., Ozawa, M.G., Snyder, E.Y., Sidman, R.L., Miller, J.H., Arap, W., and Pasqualini, R. Networks of gold nanoparticles and bacteriophage as biological sensors and cell-targeting agents. Proc Natl Acad Sci U S A 103, 1215, 2006.

48. Souza, G.R., Yonel-Gumruk, E., Fan, D., Easley, J., Rangel, R., Guzman-Rojas, L., Miller, J.H., Arap, W., and Pasqualini, R. Bottom-up assembly of hydrogels from bacteriophage and $\mathrm{Au}$ nanoparticles: the effect of cis- and trans-acting factors. PLoS One 3, e2242, 2008.

49. Souza, G.R., Molina, J.R., Raphael, R.M., Ozawa, M.G., Stark, D.J., Levin, C.S., Bronk, L.F., Ananta, J.S., Mandelin, J., Georgescu, M.-M., Bankson, J.A., Gelovani, J.G., Killian, T.C., Arap, W., and Pasqualini, R. Three-dimensional tissue culture based on magnetic cell levitation. Nat Nanotechnol 5, 291, 2010.

50. Molina, J.R., Hayashi, Y., Stephens, C., and Georgescu, M.M. Invasive glioblastoma cells acquire stemness and increased Akt activation. Neoplasia 12, 453, 2010.

51. Lee, J.S., Morrisett, J.D., and Tung, C.-H. Detection of hydroxyapatite in calcified cardiovascular tissues. Atherosclerosis 224, 340, 2012.

52. Kolonin, M.G., Souza, G.R., and Daquinag, A. Adipose tissue engineering in three-dimensional levitation tissue culture system based on magnetic nanoparticles. Tissue Eng Part C Methods 2012. [Epub ahead of print] DOI: 10.1089/ ten.tec.2012.0198.

53. Scholten, J.C.M., and Conrad, R. Energetics of syntrophic propionate oxidation in defined batch and chemostat cocultures. Appl Environ Microbiol 66, 2934, 2000. 
54. Scholten, J.C.M., Culley, D.E., Nie, L., Munn, K.J., Chow, L., Brockman, F.J., and Zhang, W. Development and assessment of whole-genome oligonucleotide microarrays to analyze an anaerobic microbial community and its responses to oxidative stress. Biochem Biophys Res Commun 358, 571, 2007.

55. Jaffe, E.A., Nachman, R.L., Becker, C.G., and Minick, C.R. Culture of human endothelial cells derived from umbilical veins. Identification by morphologic and immunologic criteria. J Clin Invest 52, 2745, 1973.

56. Hurst, D.J., Kilburn, K.H., and Baker, W.M. Normal newborn and adult human lung collagen-analysis of types. Connect Tissue Res 5, 117, 1977.

57. Orihara, K., Dil, N., Anaparti, V., and Moqbel, R. What's new in asthma pathophysiology and immunopathology? Expert Rev Respir Med 4, 605, 2010.

58. McAnulty, R.J. Models and approaches to understand the role of airway remodelling in disease. Pulm Pharmacol Ther 24, 478, 2011.

59. Ribatti, D., Puxeddu, I., Crivellato, E., Nico, B., Vacca, A., and Levi-Schaffer, F. Angiogenesis in asthma. Clin Exp Allergy 39, 1815, 2009.
60. Pageau, S.C., Sazonova, O.V., Wong, J.Y., Soto, A.M., and Sonnenschein, $\mathrm{C}$. The effect of stromal components on the modulation of the phenotype of human bronchial epithelial cells in 3D culture. Biomaterials 32, 7169, 2011.

61. Yoshikawa, T., Hamada, S., Otsuji, E., Tsujimoto, H., and Hagiwara, A. Endocrine differentiation of rat enterocytes in long-term three-dimensional co-culture with intestinal myofibroblasts. In Vitro Cell Dev Biol Anim 47, 707, 2011.

Address correspondence to: Glauco R. Souza, PhD Nano3D Biosciences

7000 Fannin St.

Suite No. 2140

Houston, TX 77030

E-mail: gsouza@n3dbio.com

Received: March 08, 2012

Accepted: January 07, 2013

Online Publication Date: February 22, 2013 